\title{
La identidad habitada: valores de la acción performativa y la palabra para la construcción de la alteridad en contextos educativos
}

\begin{abstract}
Resumen
El objeto del estudio es, primeramente, describir y analizar las aportaciones didácticas de la acción performativa a los procesos relacionales de enseñanza y aprendizaje de docentes y estudiantes de formación profesional y grado de educación infantil. Y posteriormente, realizar una comparación entre las similitudes y diferencias de las respuestas de ambos colectivos con relación a su percepción y valoración de la experiencia compartida. La propuesta artística de la obra de la artista japonesa Chiharu Shiota, basada en el encuentro y la construcción de la identidad a través de espacios y objetos simbólicos, sirve de contexto para diseñar la acción comunitaria. La metodología de investigación utilizada tiene un carácter mixto, de orientación cualitativa a través de análisis cuantitativos y también carácter exploratorio y holístico. Se desarrollaron instrumentos para la investigación consistentes en grupos de discusión de estudiantes y docentes con la finalidad de obtener información sobre los procesos relacionales que tienen lugar en la acción performativa y analizar su aplicabilidad en el ámbito educativo. Posteriormente, mediante entrevistas en grupo y a través de la palabra, se solicitó a todos los participantes que expresaran las vivencias más significativas. Las diferentes escalas de valoración ofrecen como resultado una mejora sensible del ambiente de enseñanza-aprendizaje y de convivencia en ambos colectivos, un favorecimiento de los procesos comunicativos interpersonales basados en la alteridad y la puesta en valor pedagógico de la mediación simbólica de los espacios, los objetos, el cuerpo y la palabra para representar el encuentro en la vida de relación.
\end{abstract}

\author{
María Victoria Martínez- \\ Vérez. PhD \\ Doctorado en Sociología \\ UNED-A Coruña \\ Correo electrónico: \\ vita.martinez.verez@gmail.com \\ 으 orcid.org/0000-0002-8417-5101 \\ Google Scholar
}

Antonio Montero-Seoane. $\mathrm{PhD}$

Doctorado en Educación Física /

Doctorado en Sociología

Universidad de La Coruña

Correo electrónico:

antonio.montero.seoane@udc.es

๑ orcid.org/0000-0003-4595-8530

Google Scholar

Recibido: mayo 7 de 2018

Aprobado: agosto 30 de 2019

\section{Palabras clave:}

Educación comunitaria, experiencia pedagógica, identidad, taller creativo. 


\section{Inhabited Identity: performative action and word values for the construction of otherness in educational contexts}

\begin{abstract}
The purpose of the study is, in the first place, to describe and analyze the didactic contributions of performative action to the relational processes of teaching and learning of teachers and students in the contexts of professional training and graduate studies in early childhood education. In the second place, the study makes a comparison between the similarities and differences of the answers of both groups in relation to their perception and assessment of the experience shared. The artistic proposals of the work by the Japanese artist Chiharu Shiota, based on the encounter and the construction of identity through symbolic spaces and objects serve as a context for designing community action. The research methodology used in the study is of a mixed nature with a qualitative focus through quantitative analysis and also of exploratory holistic character. Research instruments, consisting of students and teachers discussion groups were developed in order to obtain information on the relational processes that take place in performative action and analyze their applicability in the educational field. Subsequently, through group interiews and through words all participants where requested to share the most significant experiences verbally in group. The different assessment scales offer as a result a noticeable improvement of the teaching-learning environment and coexistence in both groups, a favoring of interpersonal communication processes based on otherness and a pedagogical enhancement of the symbolic mediation of spaces, objects, body and words to represent the encounter in the relationship life.
\end{abstract}

Key words:

community education, creative workshop, identity, teaching experience. 


\section{Introducción: habitar el yo y existir para el otro a través de la palabra}

Como seres contextuales e históricos, el relato de nuestra identidad comienza en una primera palabra creadora, nuestro nombre, que nos dice y está ligada al deseo de otro. Así, nacer al "ser" sólo es posible desde la espera y la acogida, ya que "reconocer al sujeto implica reconocer que el sujeto es muchos" (Madrid, 2013, p. 264). Después, el relato continúa en las circunstancias que habitan el yo y nos permiten existir para esos otros a través de la palabra, pues "sólo desde un mundo gramatical compartido es posible la relación" (Ortega, 2016, p. 253). En este sentido, nuestra realidad y todo lo que podemos decir o nos dice, se construye desde la palabra y en el símbolo. Así, el lenguaje es primero experimentado, vivido, comprendido y después compartido en la alteridad, lugar donde se producen los encuentros como instancia narrativa del "nosotros".

Y esa experiencia del otro, al significarlo como parte nuestra, nos construye y ubica en un lugar afectivo, pues es diferente ocupar un espacio que tener un lugar en los otros ya que "es necesario que todos, sujetos y comunidades, seamos capaces de descentrarnos, de extrañarnos, de mirar al "otro" que hay en mí" (Ruíz, 2010, p. 187). Y esta distinción que nos otorga ese otro, al acogernos y ser encuentro, nos hace responsables de una misma narración común pues "no nacemos como seres totalmente racionales y autónomos, sino como seres totalmente dependientes y, aún en la edad adulta, permanecemos interdependientes (Vázquez, 2010, p. 181).

De tal modo, son los vínculos afectivos que establecemos con las comunidades de pertenencia los que nos enhebran a una narración, personal y colectiva, a través del hilo del encuentro. Por eso, cada persona es un entramado infinito en el que se mezclan todas las variables hasta el punto de que cualquier hipótesis podría ser cierta en nosotros pues somos siempre seres en construcción. En ese 
entramado de la alteridad, el ámbito de la educación propone una metáfora de vida a través de valores de integración y socialización que marcarán el devenir académico y personal de los discentes.

Este acompañamiento en los procesos educativos que no separan entre quien enseña y quien aprende, debería basarse en temas surgidos de sus propuestas para que ganen confianza en sus posibilidades y se aventuren en sus campos de interés (Cabrera y de la Herrán, 2015). El docente debería potenciar la comunicación y creación de puentes que impliquen también a la institución escolar para potenciar el aprendizaje a través del deseo y la conexión con la realidad. Esta construcción dialógica estará basada en una escucha activa y sensible del "aquí y ahora" para significar el descubrimiento que permite reconocernos en la alteridad a través de la palabra y habitar ese lugar performativo que es el contexto escolar como escenario para la vida de relación.

\section{La propuesta de la acción performativa en contextos educativos}

Desde la propuesta relacional del arte como estado de encuentro (Bourriaud, 2007), los contextos educativos pueden entenderse como lugar para la producción de intersubjetividad. Así, la acción performativa posibilita los significados y relaciones que colaboran en la elaboración compartida del sentido de "Ser juntos" a través de una acción simbólica. En este entramado de identidades, la participación en una performance educativa supone realizar un ensayo para la vida y la construcción de una metáfora que resignifica los vínculos en el grupo humano de referencia, para la mejora del ambiente de convivencia y las interacciones que van más allá de lo académico. Es decir, una acción artística y educativa que precisa de la negociación, el consenso, el diálogo y el intercambio en la emoción que genera cada encuentro (Gómez, 2005). 
Partiendo de la performance como acción basada en la interacción corporal, la simbología y la concreción del lugar y el tiempo, emerge una propuesta educativa basada en un enfoque más complejo, vivencial, sistémico, creativo, dialógico, interactivo, holístico y multisensorial (Vergara, 2011). La performance es un recurso artístico-educativo que permite a los estudiantes crear colaborativamente sus propias narraciones de vida para reconocerse en ellas a través de otros y convertirse en instrumento de transformación personal y comunitaria. Como recurso didáctico permite una construcción colectiva al posibilitar que cada participante interprete cognitiva, social y afectivamente un relato común elaborado a partir de los conceptos, emociones y significados que se comparten en el contexto educativo.

Así, la acción performativa es la representación de la historia del "yo" en el encuentro lúdico con el "otro". Para Moreno (2010), la actividad artística no es un fin en sí misma, sino que actúa como mediadora, permitiendo al sujeto conectar con su identidad individual y cultural, revisar su imaginario y acceder al universo simbólico pues "permite una relación dialéctica entre lo que sabemos y lo que percibimos, entre lo aprendido y experimentado, entre el objeto y el sujeto, entre lo real y lo imaginario, entre lo sentido y lo vivido, entre la forma y los símbolos" (Moreno, 2010, p. 3). El interés de la performance educativa que se describe para este estudio es la alteridad, definida como la construcción mutua de la identidad, representada a través de una simbología compartida en la experiencia.

De esta manera, la comunidad educativa se representa en un relato colectivo basado en los procesos de vida como singular entramado de biografías y escenario de posibilidad. Para una educación sensible a las diferentes formas de convivencia es necesario desarrollar "la capacidad de ver el mundo desde la perspectiva del otro y, por lo tanto, la empatía necesaria para construir las relaciones desde la proximidad y también en la diferencia" (Argüello, Cabeza, 
Cardona, Martín \& Rodríguez, 2012, p. 407). Así, la performance educativa funciona como "acto vital de transferencia, transmitiendo saber social, memoria y sentido de identidad" (Moreno, Usán, Criado \& Santaforentina, 2013, p. 450) y, en consecuencia, se nutre de las diferentes experiencias de entendimiento mutuo que permiten a los estudiantes ser capaces de reconocer, gestionar y desarrollar sus propias expectativas, decisiones y compromisos para tomar conciencia de quienes son en relación con otros. La configuración de los espacios y tiempos concebidos para generar lo extraordinario es importante pues "el diseño del espacio físico puede acoger o dificultar los encuentros, la comunicación y los vínculos" (Errázuin-Larraín, 2015, p. 96). Así, cada espacioambiente propone la diversificación del aprendizaje para ser significativo y transformador.

La experiencia de lugar colabora entonces en la construcción de la alteridad a través de la mediación simbólica de los objetos, las imágenes, los gestos y las palabras como representación y relato del nosotros. $\mathrm{Y}$ desde esa narrativa que nos convoca en su dimensión relacional e identitaria, "se trata, fundamentalmente, de crear un mundo que proporcione sentido a la vida, a los actos humanos, a las relaciones humanas; y aquí adquiere sentido una narrativa pedagógica de la identidad" (Bernal, 2011, p. 287). También Moreno (2015) señala que la acción artística contribuye a interiorizar procesos de autoconocimiento, autoconfianza e incentivo de los procesos de socialización, integración y cooperación que faciliten la relación con las personas, los objetos y los espacios del entorno. Por todo ello, cada historia de vida resulta entonces una aportación imprescindible para hacer posible la construcción de una "arquitectura" en la que habitar la alteridad a través de los significados compartidos que suceden en la performance como celebración del encuentro en el contexto educativo. 


\section{Método}

El diseño del presente estudio tiene carácter mixto y exploratorio (Reidl, 2012, p. 38), así como holístico (Moreno, 2015). Carácter mixto por el empleo de técnicas de investigación de orientación cualitativa alrededor de un foco de interés y de unas "claves iniciales de interpretación" que "equivalen y desempeñan las mismas funciones que la teoría y las hipótesis en los análisis cuantitativos" (Ruíz Olabuénaga, 2012, pp. 56-57). Para, en un momento posterior, desarrollar un instrumento siguiendo las indicaciones de Alvira (2004) con la finalidad de obtener información sobre los procesos que tuvieron lugar en la acción y serán analizados en el apartado correspondiente.

\section{Objetivos}

El primer objetivo general, consiste en la descripción de las aportaciones de la performance a los procesos de enseñanza-aprendizaje de futuros docentes de educación infantil, que se concreta en nueve objetivos específicos:

1. Delimitar la aplicabilidad de la performance en el ámbito educativo.

2. Establecer la transversalidad de la performance para integrar diversos tipos de conocimiento.

3. Determinar la capacidad para mejorar los procesos de enseñanzaaprendizaje a través de la performance.

4. Señalar las oportunidades metodológicas de la performance en el contexto educativo.

5. Evaluar el grado de adecuación de los recursos para la intervención artística en el medio educativo.

6. Evaluar el grado de adecuación del entorno para la ejecución de la intervención artística en la escuela. 
7. Evaluar el grado de adecuación de los objetos empleados durante la acción artística.

8. Determinar el efecto de los recursos empleados respecto a los aprendizajes personales alcanzados.

9. Establecer los conceptos más representativos para los participantes en la performance.

El segundo objetivo general del estudio ha consistido en presentar las similitudes y diferencias en las respuestas de estudiantes y docentes.

En primer lugar, se realizó una revisión acerca de la aplicación de la performance en el ámbito educativo formal, en revistas publicadas en español desde el 2010 hasta el 2016 en las bases de datos Web of Science, Scopus y Latindex. Las principales conclusiones son que el uso de la performance en el contexto educativo apenas es habitual y que las pocas publicaciones que lo tratan proponen un enfoque transversal que permite vincular el pensamiento con la emoción a través del cuerpo.

\section{Población y muestra}

La selección de los participantes fue determinada por la búsqueda de información relevante para alcanzar los grados de saturación y riqueza que permitieran la consecución de los objetivos definidos. En consecuencia, se efectuó un muestreo intencional y opinático, siguiendo un criterio estratégico centrado en aquellas personas que tuvieran un conocimiento de la situación y experiencias previas en la aplicación de la performance en el ámbito educativo (Ruíz Olabuénaga, 2012).

En el estudio participaron 189 personas: 65 estudiantes del ciclo de Educación Infantil, 62 estudiantes de Grado de Educación Infantil y 62 docentes con una 


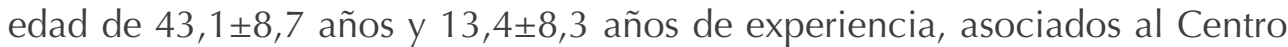
de Formación de Profesores de A Coruña y al Centro Universitario La Salle de Madrid.

\section{Instrumento}

Para la obtención de datos en esta investigación, se empleó una escala con 51 ítems agrupados en torno a los 8 objetivos descritos. Dicho instrumento se construyó "ad hoc", basándonos en los trabajos desarrollados por 2 grupos de discusión realizados en A Coruña, con 6 profesores y 7 alumnos participantes, respectivamente. Las opciones de respuesta en cada ítem fueron: 1= nada de acuerdo; $2=$ poco de acuerdo; $3=$ bastante de acuerdo y $4=$ totalmente de acuerdo. Posteriormente, en una entrevista en grupo, se solicitó a los participantes que aportasen los conceptos experimentados con un mayor significado durante la acción, siguiendo las orientaciones de Ruíz Olabuénaga (2012).

\section{Procedimiento de recogida y análisis de datos}

Al inicio de la sesión, los investigadores expusieron la obra de la artista japonesa Chiharu Shiota, quien trabaja con objetos simbólicos e instalaciones que proponen participación comunitaria y se tomó como principal referencia la obra "The Key in the Hand", Bienal de Venecia de 2015 (Figura 1). Se solicitó a los participantes (estudiantes y docentes de educación infantil) que pensaran las posibles conexiones con la formación docente. De la reflexión surgida por la obra de Shiota, aparecen los ocho objetivos que agrupan los ítems de la escala.

La justificación en la elección de la obra está basada en su propuesta para evocar temas como la ausencia y la presencia, la memoria y el olvido, el yo 
y el otro en relación con el entorno próximo y el uso de objetos simbólicos como mediadores para representar la construcción de la alteridad. Su trabajo resultó ser una interesante metáfora para dar forma a la "identidad habitada" de los participantes en la creación del relato de la acción performativa que permitió visibilizar las relaciones del grupo. Tras analizar la obra de Chiharu Shiota, los participantes simbolizaron en el aula la identidad individual y colectiva expresada a través de los mismos elementos que la artista utiliza en su obra: Ilaves, hilo rojo y la palabra escrita (Figura 2). Es importante destacar que colaboraron en la construcción de la idea de la acción, pues la integración respetuosa de las iniciativas de los estudiantes permite, por una parte, transformar las ideas del docente $y$, por otra, alejar el modelo transmisivo de enseñanza-aprendizaje (Martín y De-Juanas, 2013).

Se solicitó a los participantes que investigaran su socialización e historización a partir del origen de su nombre. Posteriormente, los investigadores propusieron la realización de una acción performativa, que tuvo lugar en octubre y noviembre de 2015 en A Coruña y Madrid, respectivamente. Fuera del aula, los participantes tejieron una red en la que cosieron palabras representativas de su propia historia de vida (Figura 3) para considerar la existencia de una humanidad vinculante que nos enhebra simbólicamente en una misma maraña de hilos como propone la artista. Tras terminar la acción, los participantes indicaron su grado de conformidad con los ítems propuestos en el instrumento y señalaron las tres palabras más representativas para contar su propia historia (Figura 4). Todos firmaron un consentimiento donde se informaba que sus respuestas se procesarían anónimamente y que los datos tendrían como fin único el propuesto. 


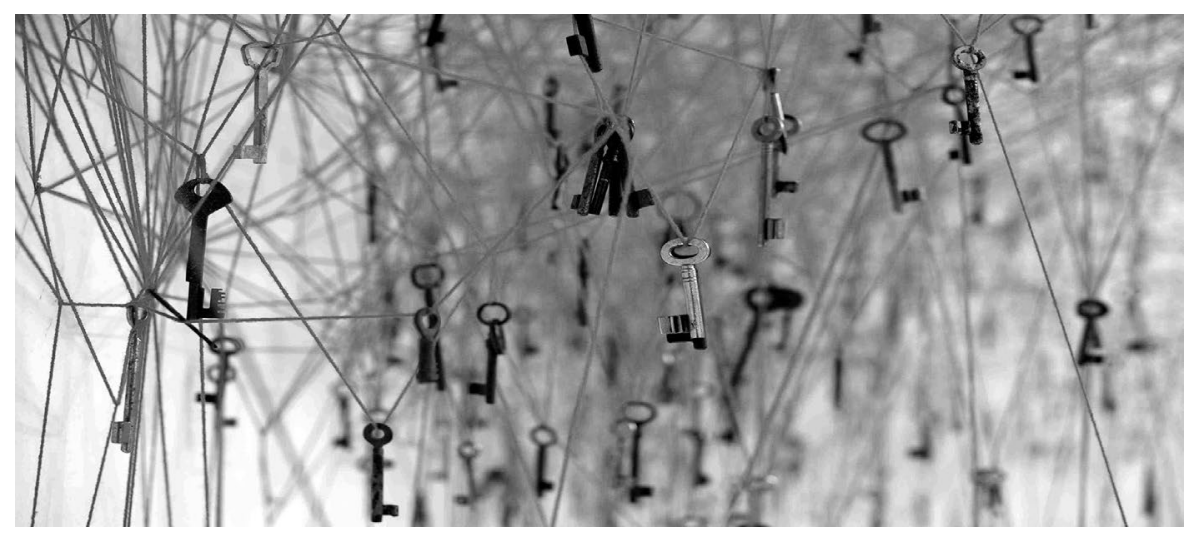

Figura 1. Chiharu Shiota: "The key in the hand". Bienal de Venecia (2015). Fuente: http://2015.veneziabiennale-japanpavilion.jp/en/

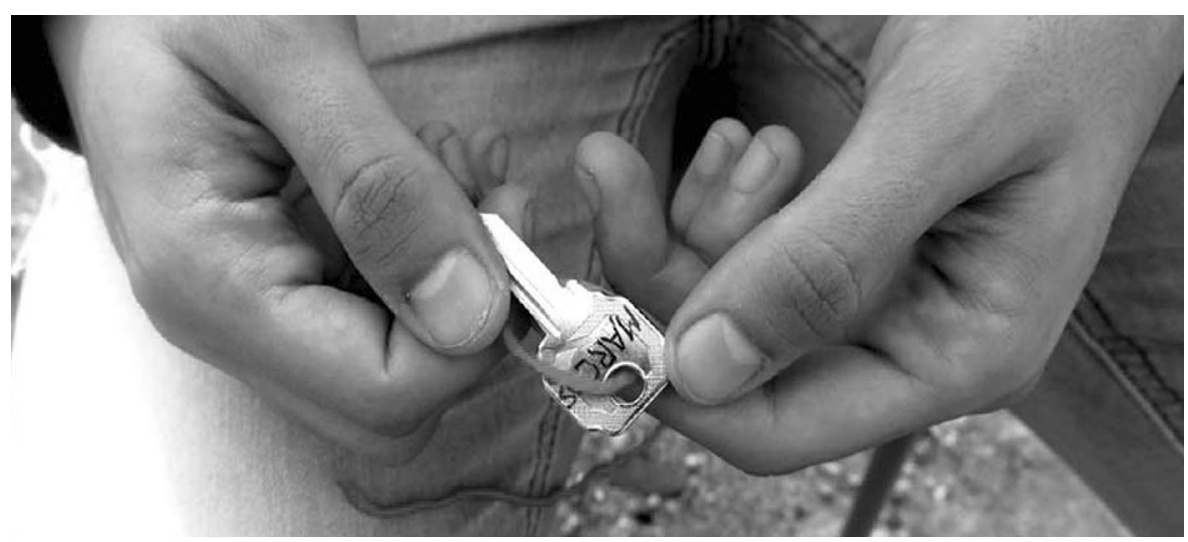

Figura 2. Representación de la identidad a través de objetos personales. Fotografía de los autores. 


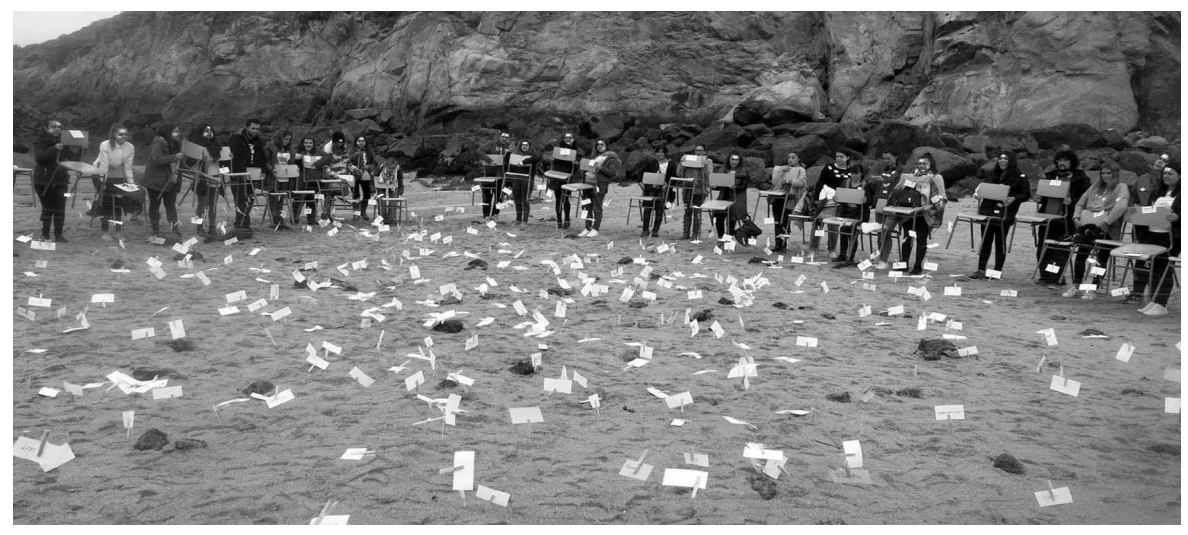

Figura 3. Creación colectiva de la red de palabras y significados. Fotografía de los autores.

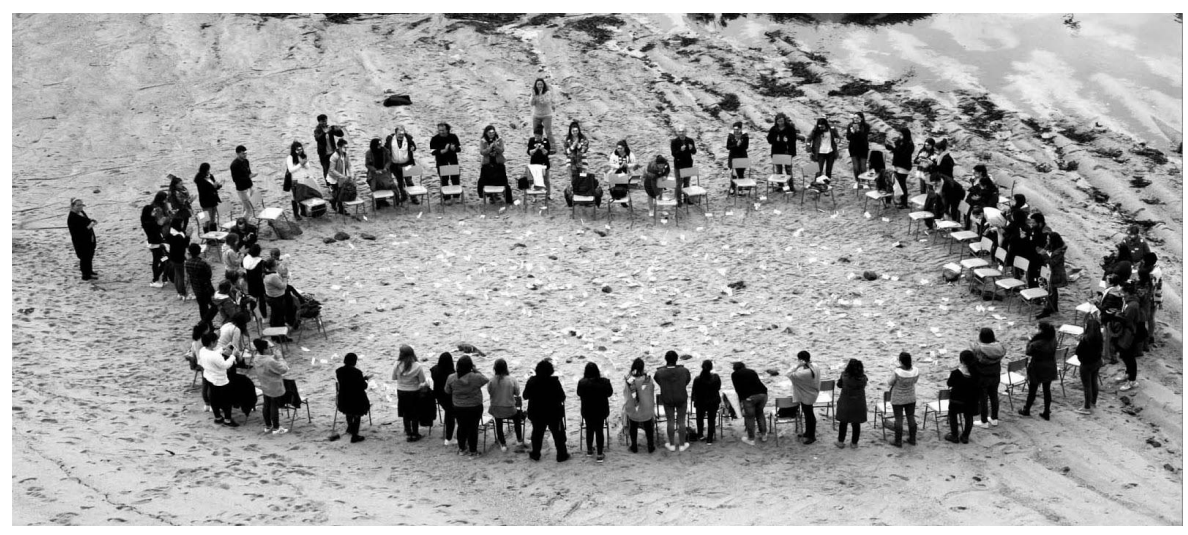

Figura 4. Reflexión y puesta en común sobre la acción performativa. Fotografía de los autores. 


\section{Resultados}

Los resultados asociados al primer objetivo general se presentarán agrupando las respuestas de los estudiantes y docentes participantes.

\section{Resultados asociados al objetivo específico (OE) 1}

Este primer objetivo específico, que denominaremos "Aplicabilidad" (tabla 1), registra frecuencias similares en los ítems propuestos, además de niveles elevados de conformidad. De este modo, se podría indicar que las acciones artísticas participativas son aplicables en diferentes etapas educativas y áreas de conocimiento, enriqueciendo al tiempo la vida personal de los participantes, ya que el acto de representar con arte, actitudes y valores, ayuda a comprender y a situar el "saber ser" en la propia vivencia. Así pues, podría decirse que la expresión artística es algo así como el lápiz del cartógrafo, no hace el mapa, pero permite trazar el dibujo.

Los conocimientos específicamente artísticos previos a la intervención, por otra parte, parecen diferir en las respuestas de estudiantes y docentes, puesto que éstos reducen el valor de los conocimientos previos para su aplicabilidad. En este sentido, la idea o metáfora cobra una importancia diferente, ya que no es la técnica o la destreza lo importante en sí, sino la capacidad de sentir y percibir lo intangible y concretarlo en una idea. 
Tabla 1. Valores de las respuestas relativas a la "aplicabilidad".

\begin{tabular}{|c|c|c|c|c|c|c|c|}
\hline \multirow{2}{*}{ Ítem } & & \multicolumn{3}{|c|}{ Estudiantes } & \multicolumn{3}{|c|}{ Docentes } \\
\hline & & $\mathrm{n}$ & Media & D.T. & $\mathrm{n}$ & Media & D.T. \\
\hline Z4 & $\begin{array}{l}\text { Las intervenciones artísticas } \\
\text { participativas son aplicables al entorno } \\
\text { escolar }\end{array}$ & 119 & 3,6 & 0,5 & 54 & 3,8 & 0,5 \\
\hline $\mathrm{Z5}$ & $\begin{array}{l}\text { Las intervenciones artísticas } \\
\text { participativas son aplicables a las } \\
\text { diferentes etapas educativas }\end{array}$ & 119 & 3,5 & 0,5 & 54 & 3,8 & 0,4 \\
\hline Z6 & $\begin{array}{l}\text { La experiencia en la que has participado } \\
\text { ha enriquecido tu vida personal }\end{array}$ & 118 & 3,3 & 0,6 & 53 & 3,1 & 0,7 \\
\hline $\mathrm{Z7}$ & $\begin{array}{l}\text { La experiencia puede ser aplicable } \\
\text { por docentes, sin unos conocimientos } \\
\text { artísticos previos }\end{array}$ & 119 & 2,8 & 0,9 & 54 & 3,3 & 0,8 \\
\hline Z8 & $\begin{array}{l}\text { La experiencia es aplicable en y por } \\
\text { cualquier materia, disciplina o área de } \\
\text { conocimiento }\end{array}$ & 119 & 3,2 & 0,6 & 54 & 3,3 & 0,7 \\
\hline
\end{tabular}

Fuente: elaboración propia.

\section{Resultados asociados al OE 2}

Los valores referidos a este objetivo específico, que denominaremos como "Transversalidad" (tabla 2), permiten apreciar valores similares en los participantes, aunque superiores entre los docentes; entre ellos, destaca el valor atribuido a la experiencia desarrollada para facilitar la integración de emoción, pensamiento y acción, así como la utilización del pensamiento alternativo, aspectos que el modelo tecnocrático de enseñanza, basado en aprendizajes cognitivos o procedimentales, así como en sistemas de control de calidad industriales, separan y desintegran, hasta el punto de reducir la escolaridad a aquello que sucede en los límites del pupitre. 
Tabla 2. Valores de las respuestas en la dimensión "Transversalidad"

\begin{tabular}{|c|c|c|c|c|c|c|c|}
\hline \multirow{2}{*}{ Ítem } & & \multicolumn{3}{|c|}{ Estudiantes } & \multicolumn{3}{|c|}{ Docentes } \\
\hline & & $\mathrm{n}$ & Media & D.T. & $\mathrm{n}$ & Media & D.T. \\
\hline Z9 & $\begin{array}{l}\text { Las acciones artísticas aplicadas al ámbito } \\
\text { educativo permiten entender los procesos } \\
\text { de enseñanza-aprendizaje desde una } \\
\text { perspectiva flexible y abierta }\end{array}$ & 119 & 3,5 & 0,5 & 54 & 3,6 & 0,5 \\
\hline Z10 & $\begin{array}{l}\text { Las acciones artísticas permiten que } \\
\text { los participantes manejen aspectos } \\
\text { transversales como la no linealidad } \\
\text { de los aprendizajes, la incertidumbre } \\
\text { respecto a las acciones propuestas y la } \\
\text { multidimensionalidad de la realidad }\end{array}$ & 117 & 3,3 & 0,6 & 54 & 3,5 & 0,6 \\
\hline Z11 & $\begin{array}{l}\text { Las acciones artísticas participativas } \\
\text { permiten integrar la emoción, el } \\
\text { pensamiento y la acción }\end{array}$ & 119 & 3,7 & 0,5 & 54 & 3,8 & 0,5 \\
\hline Z12 & $\begin{array}{l}\text { Las acciones artísticas favorecen la } \\
\text { utilización del pensamiento alternativo }\end{array}$ & 118 & 3,5 & 0,6 & 54 & 3,7 & 0,5 \\
\hline Z13 & $\begin{array}{l}\text { La experiencia artística ha sido } \\
\text { significativa en mi formación docente }\end{array}$ & 117 & 3,3 & 0,7 & 51 & 3,0 & 0,9 \\
\hline
\end{tabular}

Fuente: elaboración propia.

\section{Resultados asociados al OE 3}

La tabla 3 recoge los datos relativos a diversos mecanismos implicados en la mejora de los procesos educativos, con valores similares entre los participantes, aunque algo mayores entre los docentes. Los valores más elevados están asociados a la experimentación del concepto de grupo mediante la acción artística realizada, la mejora de los procesos comunicativos y el clima del grupo, aunque no puntúan tan alto en la capacidad para resolver conflictos interpersonales. Resulta evidente que trabajar juntos, a la vez, co-creando y sosteniendo una misma metáfora, favorece la comunicación horizontal, 
generando sentimientos de pertenencia, pero también resulta evidente que este método no es una receta universal, que ponga fin a todos los problemas socioeducativos, y es importante señalarlo, ya que la piedra filosofal, de existir tampoco solucionaría los males del universo, sino tan sólo algunos. Estamos pues, ante un camino diferente, que, a su vez, necesitará de cauces para desarrollarse y crecer.

Tabla 3. Valores de las respuestas referidos a la "Mejora de los procesos de enseñanza-aprendizaje".

\begin{tabular}{|c|c|c|c|c|c|c|c|}
\hline \multirow{2}{*}{ Ítem } & & \multicolumn{3}{|c|}{ Estudiantes } & \multicolumn{3}{|c|}{ Docentes } \\
\hline & & $\mathrm{n}$ & Media & D.T. & $\mathrm{n}$ & Media & D.T. \\
\hline Z14 & $\begin{array}{l}\text { Las intervenciones artísticas } \\
\text { participativas mejoran los procesos } \\
\text { comunicativos }\end{array}$ & 119 & 3,5 & 0,5 & 54 & 3,6 & 0,6 \\
\hline Z15 & $\begin{array}{l}\text { Las intervenciones artísticas } \\
\text { participativas favorecen la resolución } \\
\text { de conflictos interpersonales }\end{array}$ & 119 & 3,1 & 0,7 & 54 & 3,3 & 0,7 \\
\hline Z16 & $\begin{array}{l}\text { Las intervenciones artísticas } \\
\text { participativas facilitan la adquisición } \\
\text { de habilidades sociales }\end{array}$ & 119 & 3,4 & 0,6 & 54 & 3,6 & 0,6 \\
\hline Z17 & $\begin{array}{l}\text { Las intervenciones artísticas } \\
\text { participativas permiten experimentar } \\
\text { el concepto de grupo }\end{array}$ & 118 & 3,6 & 0,5 & 54 & 3,7 & 0,6 \\
\hline Z18 & $\begin{array}{l}\text { La discusión conjunta de las } \\
\text { ideas que se representan en las } \\
\text { intervenciones artísticas favorece los } \\
\text { procesos de toma de decisiones }\end{array}$ & 119 & 3,3 & 0,6 & 54 & 3,5 & 0,6 \\
\hline Z19 & $\begin{array}{l}\text { El juego de representar con otros una } \\
\text { metáfora mejora el clima del grupo y } \\
\text { la relación con los participantes }\end{array}$ & 119 & 3,5 & 0,6 & 54 & 3,6 & 0,6 \\
\hline
\end{tabular}

Fuente: elaboración propia. 


\section{Resultados asociados al OE 4}

Los datos referidos al objetivo específico denominado "Metodología" (tabla 4), registran valores similares entre estudiantes y docentes, aunque, nuevamente, con cifras algo superiores entre éstos. De dichos datos, es posible indicar una valoración altamente positiva de la experiencia vivida para favorecer el libre intercambio de experiencias entre estudiantes y docentes, la interconexión entre saberes, favorecer los procesos de reflexión personales y, todo ello, en un clima de seguridad y confianza, cuestiones éstas que inciden en la relevancia de la expresión artística como herramienta mediadora entre el aprendizaje de los valores y las actitudes del ser y la intersubjetividad del yo en relación con el grupo.

Tabla 4. Valores de las respuestas conectados con el objetivo "Metodología"

\begin{tabular}{|c|c|c|c|c|c|c|c|}
\hline \multirow{2}{*}{ Ítem } & & \multicolumn{3}{|c|}{ Estudiantes } & \multicolumn{3}{|c|}{ Docentes } \\
\hline & & $\mathrm{n}$ & Media & D.T. & $\mathrm{n}$ & Media & D.T. \\
\hline Z20 & $\begin{array}{l}\text { La utilización de las intervenciones } \\
\text { artísticas en el entorno escolar favorece la } \\
\text { interdependencia y la conectividad entre los } \\
\text { diferentes saberes }\end{array}$ & 119 & 3,3 & 0,6 & 54 & 3,5 & 0,7 \\
\hline Z21 & $\begin{array}{l}\text { La construcción de metáforas educativas } \\
\text { permite trabajar conjuntamente los } \\
\text { contenidos conceptuales, procedimentales y } \\
\text { actitudinales }\end{array}$ & 119 & 3,4 & 0,5 & 54 & 3,6 & 0,6 \\
\hline Z22 & $\begin{array}{l}\text { Alterar el ritmo escolar, modificando el } \\
\text { entorno y la metodología de aula, favorecen } \\
\text { el libre intercambio de experiencias entre } \\
\text { docentes y estudiantes }\end{array}$ & 119 & 3,6 & 0,5 & 54 & 3,7 & 0,5 \\
\hline Z23 & $\begin{array}{l}\text { La representación colectiva de las metáforas } \\
\text { favorece los procesos de reflexión personales, } \\
\text { en relación con uno mismo y con los grupos } \\
\text { de pertenencia }\end{array}$ & 119 & 3,4 & 0,6 & 54 & 3,6 & 0,6 \\
\hline Z24 & $\begin{array}{l}\text { El juego de representar con otros una } \\
\text { metáfora facilita la discusión de las ideas en } \\
\text { un clima de seguridad y confianza }\end{array}$ & 119 & 3,3 & 0,6 & 54 & 3,6 & 0,5 \\
\hline
\end{tabular}

Fuente: elaboración propia. 


\section{Resultados asociados al OE 5}

La tabla 5 muestra los resultados relativos a los "Recursos respecto a la preparación de la intervención". En ella, se pueden observar valores próximos entre los alumnos y profesores; con promedios más elevados para facilitar la comprensión de la idea de la alteridad y la propia intervención, resaltando la importancia de, por un lado, representar los conceptos abstractos, situándolos en el Yo-Otro y, por otro lado, de preparar el ambiente para que éstos se comprendan.

Tabla 5. Valores asociados al objetivo "Recursos respecto a la preparación de la intervención"

\begin{tabular}{|c|c|c|c|c|c|c|c|}
\hline \multirow{2}{*}{ Ítem } & & \multicolumn{3}{|c|}{ Estudiantes } & \multicolumn{3}{|c|}{ Docentes } \\
\hline & & $\mathrm{n}$ & Media & D.T. & $\mathrm{n}$ & Media & D.T. \\
\hline Z25 & $\begin{array}{l}\text { La información proporcionada por los } \\
\text { docentes facilitó el desarrollo de la } \\
\text { intervención }\end{array}$ & 119 & 3,3 & 0,6 & 54 & 3,6 & 0,5 \\
\hline Z26 & $\begin{array}{l}\text { La presentación de diapositivas permitió } \\
\text { entender la idea de la alteridad }\end{array}$ & 119 & 3,4 & 0,7 & 54 & 3,6 & 0,6 \\
\hline $\mathrm{Z} 27$ & $\begin{array}{l}\text { Las acciones que se realizaron sirvieron } \\
\text { para representar el concepto de } \\
\text { interrelación social }\end{array}$ & 119 & 3,5 & 0,6 & 54 & 3,5 & 0,6 \\
\hline Z28 & $\begin{array}{l}\text { La expectativa previa creada por los } \\
\text { docentes sirvió como importante } \\
\text { motivación en el grupo }\end{array}$ & 118 & 3,4 & 0,7 & 54 & 3,5 & 0,6 \\
\hline Z29 & $\begin{array}{l}\text { Los conocimientos previos sobre } \\
\text { intervenciones artísticas participativas } \\
\text { ayudan en el desarrollo }\end{array}$ & 119 & 3,3 & 0,7 & 54 & 3,3 & 0,8 \\
\hline
\end{tabular}

Fuente: elaboración propia. 


\section{Resultados asociados al OE 6}

Las respuestas a los ítems respecto a los "Recursos respecto al entorno elegido", como se aprecia en la tabla 6, registran una puntuación más elevada entre las respuestas de los estudiantes; en especial, cuando señalan que el entorno elegido para la intervención fomentó la libre expresión de las emociones, la creación de nuevos vínculos emocionales o el intercambio de experiencias personales con los compañeros. En este sentido, la elección de la playa como espacio educativo, en lugar del aula, favorece la horizontalidad de los procesos de enseñanza - aprendizaje, permitiendo a los alumnos mostrar su yo, a los compañeros y a los docentes, sin restricciones.

Tabla 6. Valores de las respuestas referidos a los "Recursos respecto al entorno elegido"

\begin{tabular}{llcccccc}
\hline \multirow{2}{*}{ Ítem } & \multicolumn{3}{c}{ Estudiantes } & & Docentes \\
\cline { 2 - 8 } Z30 & $\begin{array}{l}\text { El entorno elegido fue el escenario } \\
\text { idóneo para crear otras conexiones } \\
\text { fuera del aula }\end{array}$ & 119 & 3,6 & 0,6 & 54 & 3,3 & 0,7 \\
\hline Z31 & $\begin{array}{l}\text { El entorno elegido favoreció el } \\
\text { intercambio de experiencias personales }\end{array}$ & 119 & 3,5 & 0,6 & 54 & 3,1 & 0,8 \\
\hline Z32 & $\begin{array}{l}\text { El entorno elegido fomentó la libre } \\
\text { expresión de las emociones }\end{array}$ & 118 & 3,6 & 0,5 & 54 & 3,0 & 0,7 \\
\hline Z33 & $\begin{array}{l}\text { El entorno elegido permitió la creación } \\
\text { de nuevos vínculos emocionales con los } \\
\text { demás estudiantes }\end{array}$ & 119 & 3,3 & 0,7 & 54 & 2,9 & 0,8 \\
\hline & $\begin{array}{l}\text { El entorno elegido permitió la creación } \\
\text { de nuevos vínculos emocionales con los } \\
\text { docentes }\end{array}$ & 119 & 3,1 & 0,7 & 53 & 3,0 & 0,8 \\
\hline
\end{tabular}

Fuente: elaboración propia. 


\section{Resultados asociados al OE 7}

En la tabla 7 se expresan los valores relativos a los ítems incluidos en el objetivo denominado "Recursos respecto a la elección de los objetos". Las respuestas de estudiantes y docentes indican valores elevados y semejantes en todos los ítems analizados. En general, estos resultados indican la pertinencia de los ítems propuestos para representar de modo apropiado la idea de alteridad, favorecer la libertad de expresión emocional, el encuentro con los demás, la reflexión sobre uno mismo o comprender la experiencia como metáfora de la vida en relación. Así pues, se observa, que elegir con los profesores los elementos que representan la metáfora, permite a los alumnos sentirse protagonistas de los procesos de co-creación y representación.

Tabla 7. Valores de las respuestas centradas en los "Recursos respecto a la elección de los objetos"

\begin{tabular}{|c|c|c|c|c|c|c|c|}
\hline \multirow{2}{*}{ Ítem } & & \multicolumn{3}{|c|}{ Estudiantes } & \multicolumn{3}{|c|}{ Docentes } \\
\hline & & $\mathrm{n}$ & Media & D.T. & $\mathrm{n}$ & Media & D.T. \\
\hline Z35 & $\begin{array}{l}\text { Los objetos simbólicos elegidos } \\
\text { representaron adecuadamente la idea de } \\
\text { la alteridad }\end{array}$ & 119 & 3,4 & 0,5 & 54 & 3,4 & 0,6 \\
\hline Z36 & $\begin{array}{l}\text { Los objetos simbólicos elegidos } \\
\text { favorecieron la libertad de expresión de } \\
\text { las emociones }\end{array}$ & 119 & 3,5 & 0,6 & 54 & 3,5 & 0,7 \\
\hline Z37 & $\begin{array}{l}\text { Los objetos simbólicos elegidos } \\
\text { favorecieron el encuentro con los otros }\end{array}$ & 119 & 3,4 & 0,6 & 54 & 3,4 & 0,7 \\
\hline Z38 & $\begin{array}{l}\text { Los objetos simbólicos elegidos } \\
\text { favorecieron la reflexión respecto a uno } \\
\text { mismo }\end{array}$ & 119 & 3,5 & 0,6 & 54 & 3,4 & 0,7 \\
\hline Z39 & $\begin{array}{l}\text { Los objetos simbólicos elegidos fueron } \\
\text { importantes para entender el sentido de } \\
\text { la experiencia como metáfora de la vida } \\
\text { en relación }\end{array}$ & 118 & 3,5 & 0,6 & 54 & 3,5 & 0,6 \\
\hline
\end{tabular}

Fuente: elaboración propia. 


\section{Resultados asociados al OE 18}

La tabla 8 resume los datos relativos a los "Recursos respecto a los aprendizajes personales", donde se pueden apreciar promedios superiores entre los estudiantes; diferencia que se incrementa en ítems asociados al conocimiento o seguridad en sí mismo, aunque también se han podido observar diferencias en la toma de consciencia de las necesidades de los otros, la comprensión de conceptos con más profundidad o un desarrollo personal más equilibrado. Diferencias que podrían estar relacionadas con la etapa de desarrollo vital de los participantes, ya que el hito de la adolescencia es, precisamente, construir un Yo seguro, fortalecido e integrado, que pueda afrontar las demandas propias de la vida adulta.

Tabla 8. Valores de las respuestas "Recursos respecto a los aprendizajes personales".

\begin{tabular}{|c|c|c|c|c|c|c|c|}
\hline \multirow{2}{*}{ Ítem } & & \multicolumn{3}{|c|}{ Estudiantes } & \multicolumn{3}{|c|}{ Docentes } \\
\hline & & $\mathrm{n}$ & Media & D.T. & $\mathrm{n}$ & Media & D.T. \\
\hline Z40 & $\begin{array}{l}\text { Ser consciente de las necesidades de } \\
\text { los otros }\end{array}$ & 119 & 3,3 & 0,6 & 54 & 3,0 & 0,7 \\
\hline Z41 & $\begin{array}{l}\text { Incrementar la seguridad en mí } \\
\text { mismo/a }\end{array}$ & 119 & 3,2 & 0,7 & 54 & 2,8 & 0,9 \\
\hline Z42 & $\begin{array}{l}\text { Adquirir una mayor autonomía en la } \\
\text { toma de decisiones }\end{array}$ & 119 & 3,1 & 0,7 & 54 & 2,9 & 0,8 \\
\hline Z43 & $\begin{array}{l}\text { Entender los conceptos con mayor } \\
\text { profundidad }\end{array}$ & 119 & 3,4 & 0,7 & 54 & 3,1 & 0,6 \\
\hline Z44 & Relacionar los distintos saberes & 119 & 3,3 & 0,7 & 54 & 3,2 & 0,7 \\
\hline Z45 & $\begin{array}{l}\text { Comunicar las emociones y los } \\
\text { sentimientos, desde mí mismo, } \\
\text { respetando a los demás }\end{array}$ & 119 & 3,6 & 0,6 & 54 & 3,4 & 0,8 \\
\hline Z46 & $\begin{array}{l}\text { Establecer formas diferentes de relación } \\
\text { interpersonal }\end{array}$ & 118 & 3,4 & 0,6 & 54 & 3,4 & 0,6 \\
\hline
\end{tabular}




\begin{tabular}{clcccccc}
\hline \multirow{2}{*}{ Ítem } & \multicolumn{3}{c}{ Estudiantes } & & Docentes \\
\cline { 2 - 7 } Z47 & Decir más libremente lo que se piensa & 119 & 3,4 & 0,7 & 54 & 3,2 & 0,8 \\
\hline Z48 & Conocerme mejor a mí mismo/a & 119 & 3,5 & 0,7 & 54 & 3,2 & 0,8 \\
\hline \multirow{2}{*}{ Z49 } & $\begin{array}{l}\text { Desarrollar un autoconcepto y una } \\
\text { autoestima más equilibrados }\end{array}$ & 118 & 3,2 & 0,6 & 54 & 2,9 & 0,6 \\
\hline \multirow{2}{*}{ Z50 } & $\begin{array}{l}\text { Entender el impacto de lo que dicen } \\
\text { "los otros" sobre mi autoconcepto y } \\
\text { autoestima }\end{array}$ & 118 & 3,3 & 0,7 & 54 & 2,9 & 0,7 \\
\hline Z51 & $\begin{array}{l}\text { Descubrir "el lugar" (estatus/posición) } \\
\text { que ocupo dentro del grupo }\end{array}$ & 118 & 3,2 & 0,8 & 54 & 3,0 & 0,7 \\
\hline \multirow{2}{*}{ Z52 } & $\begin{array}{l}\text { Entender que los otros tienen } \\
\text { necesidades, emociones y afectos }\end{array}$ & 118 & 3,6 & 0,5 & 54 & 3,3 & 0,7 \\
\hline \multirow{2}{*}{ Z53 } & $\begin{array}{l}\text { Respetar las necesidades, emociones y } \\
\text { afectos de los demás }\end{array}$ & 118 & 3,6 & 0,6 & 54 & 3,4 & 0,6 \\
\hline Z54 & $\begin{array}{l}\text { Sentir claramente que pertenezco a este } \\
\text { grupo }\end{array}$ & 118 & 3,3 & 0,7 & 54 & 3,3 & 0,7 \\
\hline
\end{tabular}

Fuente: elaboración propia.

Con el objetivo de facilitar la síntesis de su experiencia en la acción artística, los participantes propusieron 28 conceptos (tabla 9). Los resultados apuntan el surgimiento de un doble eje, centrado en el "encuentro" y, resaltando aspectos funcionales por parte de los docentes y en la "comunicación" y aspectos vitales entre los estudiantes. En relación con esta cuestión de síntesis y elaboración, es importante señalar dos cuestiones. La primera, de tipo general, nos indica, que la coincidencia en la elección de las "palabras clave" de la propia experiencia forma parte de acervo vital humano que sitúa al individuo en el universo, a través de la vivencia de unos hitos compartidos. La segunda cuestión, de tipo generacional, señala que las etapas del desarrollo humano determinan la interpretación de la metáfora, ya que su significado y simbolismo, atraviesa al yo, por las heridas y los gozos abiertos a la vida, que tejen en la piel del ser humano, los procesos de memoria y recuerdo (des) afectivos. 
Martínez-Vérez, M.V. y Montero-Seoane, A. / La identidad habitada: valores de la acción performativa y la palabra para la construcción de la alteridad en contextos educativos.

Tabla 9. Frecuencias en la elección de conceptos asociados a la intervención.

\begin{tabular}{|c|c|c|c|c|}
\hline & \multicolumn{2}{|c|}{ Estudiantes } & \multicolumn{2}{|c|}{ Docentes } \\
\hline & $\mathrm{N}$ & $\%$ & $\mathrm{n}$ & $\%$ \\
\hline Comunicación & 30 & 25,2 & 15 & 27,8 \\
\hline Encuentro & 28 & 23,5 & 23 & 42,6 \\
\hline Familia & 26 & 21,8 & 9 & 16,7 \\
\hline Amor & 25 & 21,0 & 5 & 9,2 \\
\hline Afecto & 21 & 17,6 & 5 & 9,2 \\
\hline Identidad & 21 & 17,6 & 13 & 24,1 \\
\hline Vida & 20 & 16,8 & 4 & 7,4 \\
\hline Camino & 18 & 15,1 & 13 & 24,1 \\
\hline Pertenencia & 16 & 13,4 & 17 & 31,5 \\
\hline Aprendizaje & 16 & 13,4 & 4 & 7,4 \\
\hline Participación & 15 & 12,6 & 16 & 29,6 \\
\hline Nosotros & 14 & 11,8 & 7 & 13,0 \\
\hline Madre & 12 & 10,1 & 3 & 5,5 \\
\hline Fuerza & 12 & 10,1 & 3 & 5,5 \\
\hline Ser & 11 & 9,2 & 4 & 7,4 \\
\hline Yo & 11 & 9,2 & 1 & 1,8 \\
\hline Arriesgar & 10 & 8,4 & 1 & 1,8 \\
\hline Muerte & 9 & 7,6 & 0 & 0,0 \\
\hline Integración & 9 & 7,6 & 7 & 13,0 \\
\hline Transformación & 8 & 6,7 & 3 & 5,5 \\
\hline Tu nombre & 5 & 4,2 & 0 & 0,0 \\
\hline Posibilidad & 5 & 4,2 & 1 & 1,8 \\
\hline Espíritu & 4 & 3,4 & 0 & 0,0 \\
\hline Padre & 3 & 2,5 & 0 & 0,0 \\
\hline Infancia & 3 & 2,5 & 0 & 0,0 \\
\hline Cuidado & 2 & 1,7 & 0 & 0,0 \\
\hline Tú & 2 & 1,7 & 0 & 0,0 \\
\hline Red & 1 & 0,8 & 5 & 9,2 \\
\hline
\end{tabular}

Fuente: elaboración propia. 


\section{Resultados asociados al objetivo 2}

Con el fin de comparar los resultados de alumnos y profesores se ha procedido a la realización de un análisis de conglomerados jerárquico de variables (o clúster), para clasificar los ítems propuestos y que se han detallado entre las tablas número 1 y 8, en la columna "ítem". La definición seleccionada contiene, finalmente, 5 clúster para cada grupo de participantes: estudiantes y docentes. Dicha selección se efectuó con el objetivo de reducir la presencia de variables con un bajo nivel de asociación con los demás ítems propuestos. Los datos proceden de los 157 participantes que han respondido a todos los ítems $(90,8 \%)$. Las figuras 5 y 6 emplean una representación denominada dendograma, para indicar las distancias entre los ítems.

De la distribución de las variables y las respuestas de profesores y alumnos, como se puede apreciar en las figuras 5 y 6 , es posible sugerir diversas similitudes y diferencias entre docentes y estudiantes $y$, en consecuencia, orientar nuevos trabajos a partir de la selección de los grupos de ítems que nos pudieran interesar. 


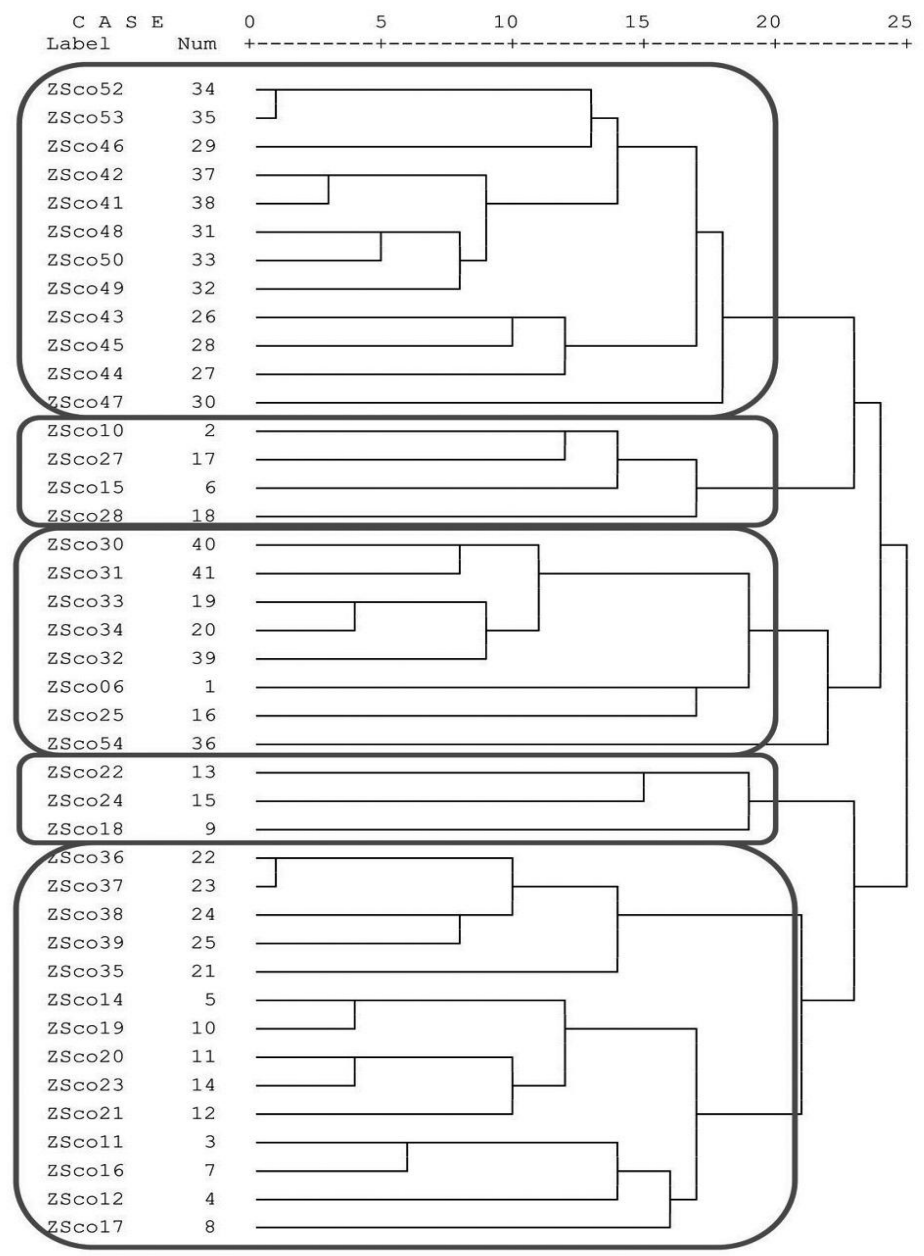

Figura 5. Clúster de variables sobre la representación artística en educación de los profesores. Fuente: elaboración propia. 


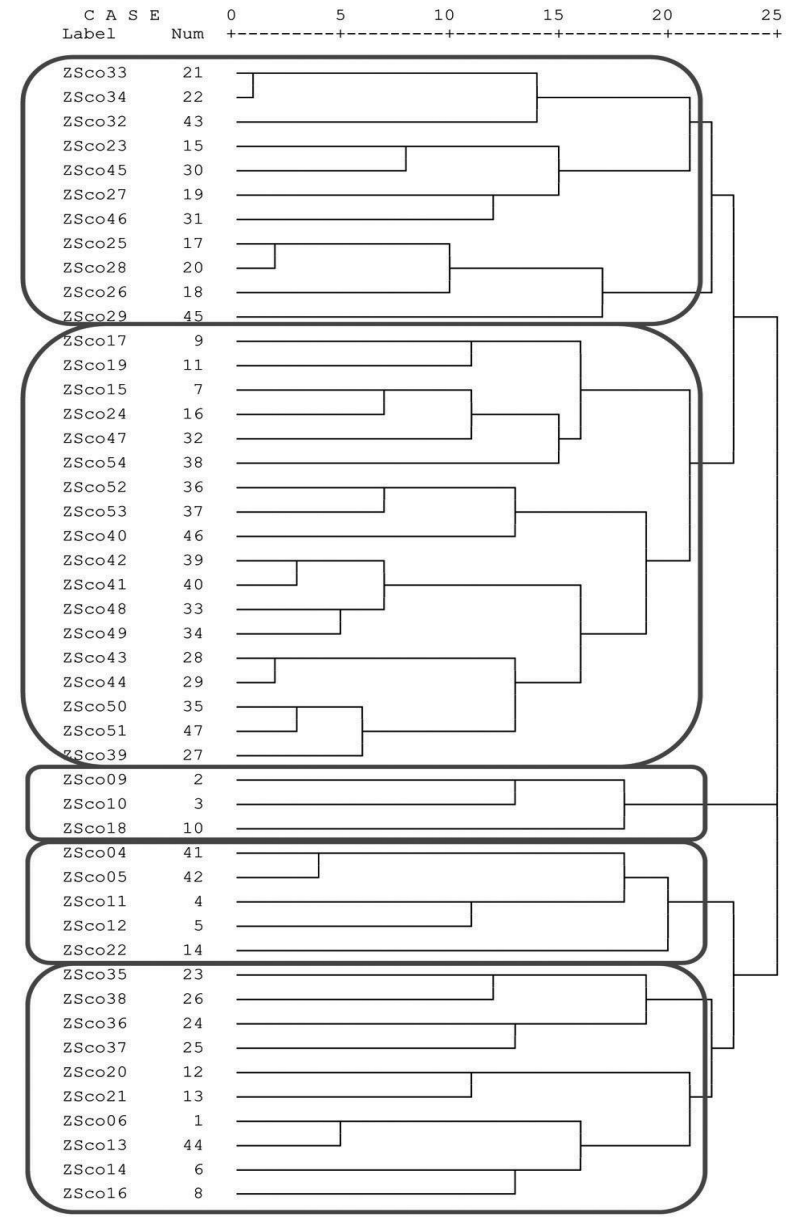

Figura 6. Clúster de variables sobre la representación artística en educación de los alumnos. Fuente: elaboración propia. 
Así, el clúster 1, similar en ambos grupos enfatiza, además, la aplicabilidad para los estudiantes y la visión de grupo e integración entre los docentes. En el clúster 2, los estudiantes acentúan la posibilidad de la acción artística como recurso útil y polivalente en varias etapas educativas, mientras que los docentes comparten con ellos la importancia del entorno y la metodología propuesta como facilitadores del libre intercambio entre docentes y estudiantes, así como la discusión conjunta para facilitar la toma de decisiones. El clúster 3 de los estudiantes insiste en las capacidades de la acción artística como promotora de procesos educativos de modo flexible y abierto o potenciadora de la transversalidad, además del ítem relativo a la mejora de la toma de decisiones mediante la discusión conjunta que aparecía en el clúster anterior entre los docentes mientras que incluyen, sobre todo, ítems relativos al efecto del entorno en la acción artística y las consecuencias positivas derivadas de ello, a lo que debería sumarse la preparación por los docentes de dicha intervención y, sobre todo, el claro sentimiento de pertenencia al grupo. El clúster 4 de los estudiantes se centra en los efectos de la metáfora y las consecuencias positivas que ha tenido la intervención artística en sí mismo, en sus capacidades de relación, sentimiento de seguridad y libertad, autoconocimiento, etc., y otros aspectos asociados al sentimiento de pertenecer al grupo y a la adjudicación de estatus dentro del mismo.

Por su parte, los docentes, conectan ítems relativos a cómo la acción performativa potencia la comprensión de los procesos educativos de modo flexible y abierto, ayuda a resolver los conflictos interpersonales o facilita la representación de conceptos de interrelación social. Finalmente, en el clúster 5 de los estudiantes se aprecian, sobre todo, ítems ya presentes en los conglomerados 3,4 y 5 de los docentes, centrados en los efectos positivos del entorno elegido y la preparación de la acción artística, la ayuda para comunicar respetuosamente emociones y sentimientos derivada, en parte al menos, del establecimiento de formas diferentes de relación interpersonal. El quinto clúster 
de los docentes concentra, sobre todo, ítems que los estudiantes clasificaban en torno a los conglomerados 4 (la mayoría) y 5 que enfatizan la percepción de mejora personal para comunicar, entender y relacionarse con otros, en la profundización e incremento de la relación entre saberes, la consecución de mayor seguridad personal y la toma de decisiones como producto de la acción artística realizada; es decir, de una autoestima y autoconcepto más equilibrado y un mejor conocimiento de sí mismo. Cuestiones que se explican desde los objetivos didácticos, siendo la perspectiva de grupo y el intercambio significativo más importante para la función tutorial-docente, y la intersubjetividad que favorece el sentimiento de pertenencia, más relevante para los alumnos que se sitúan en el último tramo de la adolescencia.

\section{Discusión}

La acción planteada parece servir a numerosos propósitos pedagógicos como la mejora de los procesos comunicativos, la resolución de conflictos interpersonales y la adquisición de habilidades sociales; aunque la puntuación más elevada entre estudiantes y docentes es la capacidad para experimentar el concepto de grupo. Por ello, si se entiende la alteridad como búsqueda del Otro, parece posible otorgar a este tipo de acciones un "referente sobre el que construir la moral" (Ruíz, 2010, p. 181). Se observa que la representación del concepto de alteridad, a través de los objetos simbólicos y el encuentro, es valorada positivamente por docentes y estudiantes. Concretamente, algunas cuestiones como el impacto de dicha representación y la interacción derivada de la acción, registran valores más elevados en los estudiantes (tabla 8), lo que podría deberse a que éstos se ven más afectados por las relaciones docenteestudiante en su autoestima y autoconcepto.

El ejercicio profesional educativo incide en unas prácticas objetivas y homogéneas, centradas en los resultados de aprendizaje que han de obtener 
los estudiantes, las cuales, además de no tener en cuenta el asombro, el deseo y la singularidad de cada persona, dificultan la generación de "espacios y tiempos que hagan posible la confrontación respetuosa y el enriquecimiento entre significados de distintas culturas" (Ruíz, 2010, p. 182)". Dichas prácticas se realizan en un espacio físico y deontológico, el aula y el currículum, pero no contemplan al otro como lugar de Ser. En este sentido, los resultados de la experiencia desarrollada y referidos a la dimensión "transversalidad", parecen indicar la posibilidad de un enfoque más flexible y abierto, multidimensional y creativo a través de experiencias que permitan intercambiar y conectar diferentes saberes en un clima de confianza para representar el concepto de alteridad.

El entorno donde transcurre la acción educativa está determinado por el espacio físico y simbólico que se construye continuamente mediante los procesos enseñanza-aprendizaje. Los resultados de la investigación indican una valoración más positiva de los estudiantes respecto a los escenarios seleccionados. Diferencias que se incrementan al afirmar que la acción performativa favoreció el intercambio de experiencias personales y la libre expresión de las emociones. Estos valores consideran el entorno como un recurso ambivalente, capaz de acoger o dificultar los encuentros, la resolución de problemas y el descubrimiento del otro para incorporar la "perspectiva del alumnado, de los docentes, apoderados y otros involucrados en el proceso" (Errázuin-Larraín, 2015, 96), superando el espacio-entorno dado e inmóvil, para recorrer otros caminos y construir otras relaciones nacidas en la confluencia de ese lugar dado y recibido.

De los resultados del estudio en relación al "yo", cabe indicar la agrupación de las respuestas de los estudiantes a diversos ítems (seguridad en sí mismo, mayor autonomía en la toma de decisiones y comprensión de los conceptos) en el cuarto cluster indicado asociado a ítems referidos al grupo, al "otro" y a 
la vida en relación; datos que parecen apuntar a una conexión estrecha entre el "yo" y el "otro", como habitantes de la propia identidad (Ruíz, 2010).

\section{Conclusiones}

Las principales conclusiones del presente artículo son:

Respecto al primer objetivo general

1.1. Docentes y estudiantes de ambos niveles educativos consideran aplicable la performance en el ámbito escolar, para representar la alteridad y la idiosincrasia del ser personal.

1.2. Las respuestas de los docentes participantes alcanzan valores más elevados en la transversalidad de la performance para la integración de emociones, pensamientos y acciones.

1.3. Los docentes registran puntuaciones más altas en todos los ítems asociados con la mejora de los procesos de enseñanza-aprendizaje: entre ellos, la experimentación del concepto de grupo, la adquisición de habilidades sociales y la resolución de conflictos interpersonales, aunque los valores más elevados corresponden a la mejora de los procesos comunicativos y del clima de grupo.

1.4. Los registros correspondientes a los docentes denotan valores más altos que los que alcanzan los estudiantes. La cifra más elevada en ambos grupos señala que la performance realizada ha favorecido el libre intercambio de experiencias entre docentes y estudiantes.

1.5. La preparación de la intervención ha sido valorada de modo similar por estudiantes y docentes en relación con la motivación, con el concepto de interrelación social y la importancia de los conocimientos previos a la misma; mientras que la facilitación del desarrollo de la intervención y la comprensión de la idea de alteridad obtuvieron valores más altos entre los docentes. 
1.6. La valoración del entorno para realizar la experiencia ha sido más elevada entre los estudiantes que entre los docentes; con mayores diferencias en los ítems referidos a la expresión de las emociones e intercambio de experiencias personales.

1.7. Los objetos simbólicos elegidos para la intervención han sido valorados de modo similar por estudiantes y profesores.

1.8. Los aprendizajes personales vivenciados han sido valorados de modo más elevado por los estudiantes, con una diferencia mayor respecto a los docentes en relación a lo que aportó para la mejoría de seguridad en sí mismo y comprender mejor el impacto de lo que dicen otros en su autoestima y autoconcepto.

1.9. Estudiantes y docentes coinciden en señalar la experiencia como un "encuentro", pero, mientras que los estudiantes señalan la "comunicación" y la "familia" como palabras clave, los docentes indican la "pertenencia" y la "participación". De ahí que se pueda establecer una orientación más "intimista" entre los estudiantes y más "operativa" entre los docentes participantes.

Respecto al objetivo general 2

2.1. Los conglomerados presentados indican enlaces similares entre las variables presentadas. Dicha similitud no puede ocultar diferencias en aspectos como la aplicabilidad de la experiencia realizada en diferentes etapas educativas o la comprensión de los procesos educativos de un modo más abierto y flexible que se integran en los clúster de los docentes mientras que no aparecen entre los estudiantes.

En definitiva, la realización de acciones artísticas con docentes o estudiantes ha de considerarse una limitación a la vez que una oportunidad para aplicar estrategias con estudiantes de las etapas del sistema educativo formal para, posteriormente, poder generalizar los conocimientos generados y 
las experiencias vividas. Por otra parte, parece necesario profundizar en las experiencias sensoriales y emocionales que se derivan de las acciones performativas, así como la conexión entre los diferentes tipos de aprendizajes y significados.

\section{Referencias}

Alvira, F. (2004). La encuesta: una perspectiva general metodológica. Madrid: CIS.

Argüello, A., Cabeza Ó, Cardona, R., Hernández, M. y Rodríguez, D. (2012). Del modelo de desarrollo económico al paradigma del desarrollo humano: una apuesta al papel del arte y las Humanidades en el pensamiento de Martha Nussbaum. Revista Complutense de Educación, 23 (2), 401-425. DOI: https:// doi.org/10.5209/rev_RCED.2012.v23.n2.40035

Bernal, A. (2011). Postmodernización y Educación. Notas para el debate de una narrativa pedagógica centrada en la identidad. Educación XX1, 14 (2), 285302. DOI: https://doi.org/10.5944/educxx1.14.2.255

Bourriaud, N. (2007). Estética relacional. Buenos Aires: Adriana Hidalgo.

Cabrera, J. y De La Herrán, A. (2015). Creatividad, complejidad y formación: un enfoque transdisciplinar. Revista Complutense de Educación, 26(3), 505-526. DOI: https://doi.org/10.5209/rev_RCED.2015.v26.n3.43876

Errázuin-Larraín, L. (2015). Calidad estética en el entorno escolar el (f)actor invisible. Arte, Individuo y Sociedad, 27 (1), 81-100. DOI: https://doi.org/10.5209/rev_ ARIS.2015.v27.n1.43861

Gómez, J.R. (2005). Posibilidades educativas de la performance en la enseñanza secundaria. Arte, Individuo y Sociedad, 17, 115-132. Recuperado a partir de https://revistas.ucm.es/index.php/ARIS/article/view/ARIS0505110117A 
Madrid, M. (2013). Investigando con otros: la investigación en educación artística como praxis de la diferencia. Arte, Individuo y Sociedad, 25 (2), 261-270. DOI: https://doi.org/10.5209/rev_ARIS.2013.v25.n2.39023

Martín, R. y De-Juanas, A. (2013). La valoración de los maestros sobre la utilización didáctica de las ideas de los alumnos. Revista Complutense de Educación, 24 (2), 267-285. DOI: https://doi.org/10.5209/rev_RCED.2013.v24.n2.42079

Moreno, A. (2010). La mediación artística: un modelo de educación artística para la intervención social a través del arte. Revista Iberoamericana de Educación / Revista Ibero-americana de Educaçao, 2 (52) - 25/03/10. DOI: 10.35362/ rie5221797

Moreno, A. (2015). Arte como herramienta social y educativa. Revista Complutense de Educación, 26 (2), 315-329. DOI: https://doi.org/10.5209/rev_RCED.2015. v26.n2.43060

Moreno, A., Usán, S., Criado, C. y Santaforentina, A. (2013). Transitando identidades. La mediación artística en el proceso de rehabilitación de personas con problemas de adicciones. Cuadernos de Trabajo Social, 26 (2), 445-454. DOI: https://doi.org/10.5209/rev_CUTS.2013.v26.n2.40582

Ortega, P. (2013). La pedagogía de la alteridad como paradigma de la educación intercultural. Revista Española de Pedagogía, LXXI (256), 401-422. DOI: http:// dx.doi.org/10.14201/teoredu30195116

Ortega, P. (2016). La ética de la compasión en la pedagogía de la alteridad. Revista Española de Pedagogía, LXXIV (264), 443-264.

Recuperado de: https://revistadepedagogia.org/lxxiv/no-264/la-etica-de-lacompasion-en-la-pedagogia-de-la-alteridad/101400001907/

Reidl, L.M. (2012). El diseño de investigación en educación: conceptos actuales. Metodología de Investigación en Educación Médica, 1 (1), 35-39. Recuperado de: http://riem.facmed.unam.mx/ojs/index.php/riem/article/view/399/370 
Ruíz, C. (2010). La educación en la sociedad postmoderna: Desafíos y oportunidades. Revista Complutense de Educación, 21 (1), 173-188. Recuperado de: https:// revistas.ucm.es/index.php/RCED/article/view RCED1010120173A

Ruíz Olabuénaga, J.I. (2012). Metodología de la investigación cualitativa. Bilbao: Universidad de Deusto.

Shiota, C. (2015). "The key in the hand". Bienal de Venecia. Recuperado de http://2015.veneziabienale-japanpavilion.jp/en/

Vázquez, V. (2010). La perspectiva de la ética del cuidado: una forma diferente de hacer educación. Educación XX1, 13 (1), 177-197. DOI: https://doi. org/10.5944/educxx1.13.1.282

Vergara, J.J. (2011). Prácticas de Perfomance y Educación. Universidad de Murcia. Recuperado de http://www.colectivoenaccion.es/Joomla15/articulos-deeducacion/110?task=view.

Como citar: Martínez-Vérez, M.V. y Montero-Seoane, A. (2020). La identidad habitada: valores de la acción performativa y la palabra para la construcción de la alteridad en contextos educativos. Revista KEPES, 17 (21), 361-394. DOI: 10.17151/kepes.2020.17.21.13 